\title{
FENÓLICOS TOTAIS E CAPACIDADE ANTIOXIDANTE IN VITRO DE POLPAS DE FRUTOS TROPICAIS ${ }^{1}$
}

\author{
LUANNE MORAIS VIEIRA², MARIANA SÉFORA BEZERRA SOUSA ${ }^{3}$, \\ JORGE MANCINI-FILHO ${ }^{4}$, ALESSANDRO DE LIMA ${ }^{5}$
}

RESUMO - O consumo de frutos e suas polpas tem sido muito recomendado por seu valor nutricional, alto teor de fibras, vitamina $\mathrm{C}$ e carotenoides. Trabalhos recentes têm apontado esses alimentos como fontes de compostos fenólicos com ação antioxidante, portanto sequestradores de radicais livres, com ação protetora contra o surgimento e/ou desenvolvimento de processos degenerativos que conduzem a doenças crônicas não transmissíveis. Devido à crescente comercialização e consumo de polpas de frutas no Brasil, especialmente na cidade de Teresina-Piauí, este trabalho selecionou um grupo de polpas de frutos de elevado consumo local para avaliação do teor de fenólicos totais e da atividade antioxidante in vitro pelo método de captura de radicais livres: DPPH (radical 1,1-diphenil-2-picrilhydrazil) e ABTS (radical 2,2'azinobis(3-ethylbenzthiazoline-6-sulfonic acid)). Os frutos selecionados foram: Acerola (Malpighia emarginata DC.), Bacuri (Platonia insignis Mart.), Cajá (Spondias mombin L.), Caju (Anacardium occidentale), Goiaba (Psidium guajava) e Tamarindo (Tamarindus indica L.). Os teores de fenólicos totais encontrados nas polpas congeladas destes frutos exibiram quantidades relevantes de polifenóis, destacando-se a polpa de acerola com 835,25 $\pm 32,44$ e 449,63 $\pm 10,24 \mathrm{mg} / 100 \mathrm{~g}$ nos extratos aquosos e hidroalcoólicos, respectivamente, seguido pela polpa de caju com 201,61 $\pm 19,15$ e 165,07 $\pm 4,10 \mathrm{mg} / 100 \mathrm{~g}$. As polpas de bacuri e tamarindo foram as que apresentaram os menores teores de fenólicos totais. Com relação à atividade antioxidante in vitro, os melhores resultados foram encontrados para os extratos aquosos e hidroalcoólicos das polpas de acerola, caju e goiaba. A capacidade antioxidante destas polpas $\left(\mathrm{EC}_{50} \mathrm{em} \mu \mathrm{g} / \mathrm{mL}\right)$ variou de 24,42 a 413,36 e de 1,74 a 259,18 para os extratos aquosos e hidroalcoólicos, respectivamente. Utilizando o radical ABTS, a atividade antioxidante para essas mesmas polpas de frutas apresentou valores TEAC que variaram de 3,69 $\pm 0,209$ a $0,052 \pm 0,013(\mathrm{mM}$ TROLOX/g de polpa). Foi observado existir uma correlação direta entre a quantidade de fenólicos totais e a atividade antioxidante nas polpas avaliadas.

Termos para Indexação: atividade antioxidante, ABTS, DPPH e frutos.

\section{TOTAL PHENOLICS AND ANTIOXIDANT CAPACITY "IN VITRO" OF TROPICAL FRUIT PULPS}

\begin{abstract}
The consumption of fruit, pulps has been highly recommended for its nutritional value, high-fiber, vitamin $\mathrm{C}$ and carotenoids. Recent studies have demonstrated these foods as sources of phenolic compounds with antioxidant activity, therefore, free radical scavengers, with a protective effect against the emergence and development or degenerative processes that lead to chronic diseases. Due to the increasing commercialization and consumption of fruit pulp in Brazil, especially in the city of Teresina, Piauí, this study selected a group of fruit pulp of high local consumption to evaluate the total phenolic content and antioxidant activity in vitro by the method of capture of free radicals: DPPH (1,1-radical diphenil-2-picrilhydrazil) and ABTS (2,2 'azinobis-(3-ethylbenzthiazoline-6-sulfonic acid)). The fruit were selected Acerola (Malpighia emarginata DC.) Bacuri (Platonia insignis Mart.) Caja (Spondias mombin L.), Cashew (Anacardium occidentale), Guava (Psidium guajava) and Tamarind (Tamarindus indica L.). The levels of phenolics found in the frozen pulp of fruit exhibited relevant quantities of polyphenols, especially if the pulp with $835.25 \pm 32.44$ and 449.63 $\pm 10.24 \mathrm{mg} / 100 \mathrm{~g}$ in hydroalcoholic and aqueous extracts, respectively followed by cashew pulp with $201.61 \pm 19.15$ and $165.07 \pm 4.10 \mathrm{mg} / 100 \mathrm{~g}$. The pulp of tamarind bacuri showed the lowest levels of total phenolics. Regarding the antioxidant activity in vitro, the best results were found for aqueous and ethanolic of pulp of acerola, cashew and guava. The antioxidant activity of these pulps $\left(\mathrm{EC}_{50} \mathrm{in} \mathrm{mg} / \mathrm{mL}\right)$ ranged from 24.42 to 413.36 and 1.74 to 259.18 for the hydroalcoholic and aqueous extracts, respectively. Using the ABTS radical, the antioxidant activity for these same fruit pulps showed that TEAC values ranged from $3.69 \pm 0.209$ to $0.052 \pm 0.013(\mathrm{mM}$ Trolox / g of pulp). We observed that a direct correlation exists between the amount of total phenolics and antioxidant activity in the analyzed pulps.
\end{abstract}

Index Terms: antioxidant activity, ABTS, DPPH and fruit.

\footnotetext{
${ }_{1}^{1}$ (Trabalho 183-10). Recebido em: 30-07-2010. Aceito para publicação em: 21-12-2010.

${ }^{2}$ Tecnóloga em Alimentos. Mestranda em Alimentos e Nutrição. Universidade Federal do Piauí - UFPI, Câmpus Universitário Petrônio Portela, s/n 64049-550. Teresina - Piauí - Brasil, E-mail: luanne.morais@gmail.com

${ }^{3}$ Tecnóloga em Alimentos. Instituto Federal de Educação, Ciência e Tecnologia do Piauí (IFPI), Praça da Liberdade 1597, Teresina-PI, 6404-000. E-mail: mariansefora@yahoo.com.br

${ }^{4}$ Professor Titular do Departamento de Alimentos e Nutrição Experimental da Faculdade de Ciências Farmacêuticas da Universidade de São Paulo. E-mail: jmancini@usp.br

${ }^{5}$ Nutricionista. Doutor em Ciências dos Alimentos pela Universidade de São Paulo - USP. Professor Adjunto do Instituto Federal de Educação, Ciência e Tecnologia do Piauí - IFPI. Avenida Pedro Freitas, 1020, São Pedro, Teresina, Piauí, CEP: 64001-010. E-mail: alessandro@cefetpi.br.
} 


\section{INTRODUÇÃO}

Com uma extensão territorial de 8.512.965 $\mathrm{km}^{2}$, o Brasil produz cerca de 43 milhões de toneladas de frutos anuais, sendo o terceiro maior produtor mundial, ultrapassado apenas pela China e Índia, primeiro e segundo maiores produtores de frutos, respectivamente. A fruticultura brasileira concentra-se principalmente nas regiões Nordeste, Sudeste e Sul, onde as condições de clima são bem distintas, permitindo o cultivo de um número variado de plantas frutíferas, tanto as tropicais como as de clima temperado (TEIXEIRA, 2009).

O Brasil, por essas características naturais, destaca-se como um dos grandes produtores mundiais de frutas in natura, porém, por serem perecíveis, grande parte dessas frutas sofre deterioração em poucos dias, tendo sua comercialização dificultada, especialmente a longas distâncias. A produção de polpas de frutas congeladas tem-se destacado como uma importante alternativa para o aproveitamento dos frutos durante a safra, permitindo a estocagem das polpas fora da época de produção dos frutos in natura (SANTOS et al., 2008).

O valor nutricional é um dos principais fatores que conduzem ao interesse crescente pelo consumo de frutos e suas polpas. Estas têm sido altamente recomendadas, pela riqueza em carboidratos, fibras, minerais, vitamina $\mathrm{C}$, carotenoides, substâncias fenólicas, substâncias sulfuradas, dentre outras, e pela ação antioxidante, que contribuem para manter o equilíbrio entre a produção e a eliminação de espécies reativas de oxigênio e outros compostos relacionados, inibindo e reduzindo as lesões causadas pelos radicais livres nas células (MAIA, 2007).

Atualmente, pesquisas têm demonstrado que os compostos fenólicos são fitoquímicos que apresentam grande interesse nutricional por contribuir para a saúde humana, devido à capacidade anticarcinogênica e antimutagênica (HEIN, 2002; SHAHIDI et al., 2007). Sabendo que a prevenção é uma estratégia mais eficaz que o tratamento para doenças crônicas, um constante fornecimento de vegetais contendo fitoquímicos benéficos à saúde, além da nutrição básica, é essencial fornecer um mecanismo de defesa que reduza o risco de doenças crônicas em seres humanos (PÉREZ-JIMÉNEZ, 2008).

Devido à crescente comercialização e consumo de polpas de frutas no Brasil, este trabalho visou a avaliar as propriedades antioxidantes, através da quantificação dos fenólicos totais e da atividade antioxidante in vitro das polpas de Acerola (Malpighia emarginata DC.), Bacuri (Platonia insignis Mart.), Cajá (Spondias mombin L.), Caju (Anacardium oc- cidentale), Goiaba (Psidium guajava) e Tamarindo (Tamarindus indica L.).

\section{MATERIAL E MÉTODOS}

\section{Material}

As amostras foram doadas por uma indústria de polpa de fruta, localizada na cidade de Teresina Piauí. Foram coletados de uma única vez $3 \mathrm{~kg}$ de cada polpa de fruta e imediatamente foram transportadas em caixas isotérmicas ao Laboratório de Alimentos do Instituto Federal de Educação Tecnológica do Piauí - IFPI, onde foram armazenadas a $-18^{\circ} \mathrm{C}$ até o momento das análises. As polpas de fruta congeladas utilizadas neste trabalho foram: Acerola (Malpighia emarginata DC.), Bacuri (Platonia insignis Mart.), Cajá (Spondias mombin L.), Caju (Anacardium occidentale), Goiaba (Psidium guajava) e Tamarindo (Tamarindus indica L.).

\section{Métodos}

Preparação dos extratos de polpa de fruta

Os extratos das polpas de frutas foram obtidos utilizando água destilada e álcool etílico P.A. (95\%) para a obtenção do extrato aquoso e hidroalcoólico (LIMA, 2008). Para a preparação dos extratos, foram utilizados $50 \mathrm{~g}$ de polpa de fruta e $100 \mathrm{~mL}$ de água destilada (1:2). A mistura foi homogeneizada durante 1 hora, em frascos Erlenmeyer, usando o agitador magnético. Depois, a mistura foi centrifugada a $3.000 \mathrm{rpm}$ por 5 minutos, o sobrenadante foi armazenado em vidro âmbar sob refrigeração a $\pm 8^{\circ} \mathrm{C}$ até o momento das análises. A preparação do extrato hidroalcoólico seguiu a mesma metodologia, substituindo-se o solvente extrator pela solução composta por $80 \mathrm{~mL}$ de água destilada e $20 \mathrm{~mL}$ de álcool etílico P.A.

\section{Determinação dos fenólicos totais}

A determinação dos fenólicos totais seguiu a metodologia descrita por Swain e Hills (1959). Do extrato aquoso e hidroalcoólico de cada amostra, tomou-se $0,5 \mathrm{~mL}$ em tubo de ensaio e adicionaram-se $8 \mathrm{~mL}$ de água destilada e $0,5 \mathrm{~mL}$ do reagente Folin Ciocalteau. A solução foi homogeneizada e, após $3 \mathrm{~min}$, acrescentou-se $1 \mathrm{~mL}$ de solução saturada de carbonato de sódio $\left(\mathrm{Na}_{2} \mathrm{CO}_{3}\right)$. Decorrida 1 hora de repouso, foram realizadas as leituras em triplicata das absorbâncias em espectrofotômetro (Coleman $33 \mathrm{D})$ a $720 \mathrm{~nm}$. Utilizou-se como padrão o ácido gálico, nas concentrações de $2 ; 5 ; 10 ; 15$ e $20 \mu \mathrm{g} /$ $\mathrm{mL}$, para construir uma curva de calibração. A partir da equação da reta obtida, realizou-se o cálculo do 
teor de fenólicos totais, expresso em mg de ácido gálico/100 g de amostra.

Atividade antioxidante in vitro.

Método de captura de radicais $\mathrm{DPPH}^{\bullet}(2,2$ difenil-1-picril-hidrazil)

Este método tem por base a redução do radical $\mathrm{DPPH}^{\bullet}$, que ao fixar um $\mathrm{H}^{*}$ (removido do antioxidante em estudo), leva a uma diminuição da absorbância. Para a análise das amostras, adicionou-se a 1,5 mL da solução metanólica de $\mathrm{DPPH}^{\bullet}\left(6 \times 10^{-5} \mathrm{M}\right)$ uma alíquota de $0,5 \mathrm{~mL}$ das amostras contendo diferentes concentrações de cada extrato. As leituras foram realizadas em espectrofotômetro (Coleman 33 D) a 517 nm, após $2 ; 5 ; 10$ e 20 minutos do início da reação. Todas as determinações foram realizadas em triplicata e acompanhadas de um controle (sem antioxidante). A queda na leitura da absorbância das amostras foi correlacionada com o controle, estabelecendo-se a porcentagem de descoloração do radical $\mathrm{DPPH}^{\bullet}$, conforme a fórmula abaixo:

$\%$ proteção $=\left[\left(\mathrm{Abs}_{\text {controle }}-\mathrm{Abs}_{\text {branco }}\right) / \mathrm{Abs}_{\text {controle }}\right] \times 100$

Para o cálculo dos valores de $\mathrm{EC}_{50}$ (concentração do extrato necessário para reduzir $50 \%$ do radical DPPH) dos distintos extratos, foi calculada a atividade antioxidante em diferentes concentrações, de forma a traçar uma curva linear entre a capacidade antioxidante do respectivo extrato e sua concentração. Esses dados foram submetidos a uma regressão linear e obtida uma equação da reta para o cálculo do $\mathrm{EC}_{50}$ (BRAND-WYLLIANS et al., 1995).

Método do radical $\mathrm{ABTS}^{\cdot+}$ (2,2'azinobis-(3ethylbenzthiazoline-6-sulfonic acid))

Para a determinação da atividade antioxidante pelo método do radical $\mathrm{ABTS}^{\cdot+}$, usou-se a metodologia descrita por RE et al. (1999). Inicialmente, foi formado o radical $\mathrm{ABTS}^{\cdot+}$, a partir da reação de $7 \mathrm{mM}$ de ABTS com 2,45 mM de persulfato de potássio, os quais foram incubados à temperatura ambiente $\mathrm{e}$ na ausência de luz, por 16 horas. Transcorrido esse tempo, a solução foi diluída em etanol até a obtenção de uma solução com absorbância de 0,70 $( \pm 0,01)$. Para realizar as análises, foram adicionados $40 \mu \mathrm{L}$ da amostra diluída a $1960 \mu \mathrm{L}$ da solução contendo o radical e determinou-se a absorbância em espectrofotômetro (Coleman $33 \mathrm{D}$ ) a $734 \mathrm{~nm}$, após 20 minutos de reação. Como solução-padrão, usou-se o antioxidante sintético Trolox nas concentrações de 100; 200; 400; 800 e $1.000 \mu \mathrm{M}$ em etanol. Todas as leituras foram realizadas em triplicata, e os resultados foram expressos em $\mathrm{mM}$ de Trolox por grama de polpa de fruta.

\section{Análise estatística}

Os resultados foram expressos como média \pm desvio-padrão. Para a comparação das médias aritméticas, empregaram-se a análise de variância (ANOVA) e o teste de Tukey, usando o software Prisma 4.0 (GraphPad). Adotou-se o nível de significância de $5 \%$ de probabilidade $(\mathrm{p}<0,05)$. Para o estudo das correlações lineares das atividades antioxidantes pelo método de captura de ABTS e DPPH, foi utilizado o programa Origin 5.0.

\section{RESULTADOS E DISCUSSÃO}

Os resultados do conteúdo de fenólicos totais das seis polpas congeladas de frutas analisadas encontram-se apresentados na Tabela 1. Verifica-se que todas as polpas de frutas apresentaram quantidades relevantes de fenólicos totais, com destaque para a polpa de acerola com $835,25 \pm 32,44 \mathrm{mg} / 100$ $\mathrm{g}$ de fenólicos totais para o extrato aquoso e 449,63 $\pm 10,24 \mathrm{mg} / 100 \mathrm{~g}$ para o extrato hidroalcoólico, exibindo o mais elevado teor destes constituintes, entre as polpas estudadas, sendo estatisticamente diferentes das demais $(p<0,05)$. Depois da polpa de acerola, a polpa de caju apresentou os valores de $201,61 \pm 19,15$ e $165,07 \pm 4,10 \mathrm{mg} / 100 \mathrm{~g}$ de fenólicos totais, para os extratos aquoso e hidroalcoólico, respectivamente. A polpa de goiaba apresentou valores intermediários de compostos fenólicos totais, $104,76 \pm 4,39$ e $20,2 \pm 1,95$ (Tabela 1 ), e as polpas de bacuri com 10,35 $\pm 0,42$ e 7,23 $\pm 0,08 \mathrm{mg} / 100$ g e de tamarindo com $23,57 \pm 0,36$ e $23,35 \pm 0,21$ $\mathrm{mg} / 100 \mathrm{~g}$ para os extratos aquoso e hidroalcoólico, respectivamente, apresentaram os menores valores desse constituinte.

Os teores de fenólicos totais das polpas congeladas de frutas determinadas neste estudo foram superiores aos relatados por alguns autores, como Gonçalves (2008), que quantificou 8,1 e 7,1 $\mathrm{mg} / 100 \mathrm{~g}$ de fenólicos totais para polpas congeladas de bacuri e tamarindo, respectivamente, fornecidos por produtores da região Amazônica, e Kuskoski et al. (2006) quantificaram de 83,0 e $580,0 \mathrm{mg} / 100 \mathrm{~g}$ de fenólicos totais em polpas congeladas de goiaba e acerola, respectivamente, provenientes do comércio de Florianópolis - SC.

As discrepâncias nos valores de teores dos compostos fenólicos, segundo Soares (2008), podem ser influenciadas por diversos fatores, tais quais maturação, espécie, práticas de cultivo, origem geográfica, estágio de crescimento, condições de colheita e processo de armazenamento das frutas. A 
peculiaridade metodológica relacionada ao solvente extrator e aos fenólicos usados como padrão para a quantificação dos compostos fenólicos também pode contribuir para as diferenças observadas.

Comparando a eficiência do solvente de extração, a partir da Tabela 1, pode-se ainda constatar que a água pura apresentou melhor poder extrator para os compostos fenólicos das polpas de frutas, se comparado com a solução hidroalcóolica (19\%). Evidencia-se, portanto, que a maior parte dos compostos fenólicos dessas frutas apresenta maior polaridade, portanto são mais hidrossolúveis. Esses achados são corroborados por Wu et al. (2004), que, ao avaliarem o conteúdo de polifenóis de frutas consumidas nos Estados Unidos, observaram que a fração hidrofílica possuía uma quantidade bem superior desses constituintes que a porção lipofílica.

A atividade antioxidante, utilizando o método de captura dos radicais DPPH, dos extratos aquosos e hidroalcoólicos das polpas de frutas avaliadas neste estudo está apresentada na Tabela 2. Os resultados foram expressos em $\mathrm{EC}_{50}$ (concentração de extrato em $\mu \mathrm{g} / \mathrm{mL}$ capaz de reagir com $50 \%$ do radical presente na solução de DPPH). Portanto, quanto menor o valor do $\mathrm{EC}_{50}$, maior será a atividade antioxidante do extrato analisado.

Como se pode observar na Tabela 2, a polpa de acerola apresentou a maior atividade antioxidante com valores de $\mathrm{EC}_{50}$ de 24,42 para o extrato aquoso e de 1,74 para o extrato hidroalcoólico, sendo seguido pelo extrato hidroalcoólico da goiaba com um $\mathrm{EC}_{50}$ de 19,69. A polpa de bacuri apresentou a menor atividade antioxidante com um $\mathrm{EC}_{50}$ de 4.700,24 e 2.356,96 para o extrato aquoso e hidroalcoólico, respectivamente. Resultados semelhantes aos obtidos por Melo et al. (2008), que constataram que os extratos aquosos das polpas de acerola e goiaba possuem forte atividade em sequestrar o radical DPPH. Esses autores observaram ainda que as polpas de caju, mamão e pinha possuem fraca capacidade em degradar o radical DPPH.

Nas Figuras 1 a 12, podem ser visualizadas as curvas cinéticas de degradação do radical DPPH pelos diferentes extratos, em diferentes concentrações, das polpas de frutas utilizadas neste estudo. Percebe-se, a partir dessas figuras, que cada extrato possui um comportamento distinto de acordo com a concentração testada. Os extratos da polpa de acerola e goiaba apresentaram forte capacidade antioxidante nos primeiros 5 minutos de reação, com expressiva redução do radical DPPH. Os extratos de polpa de cajá e caju apresentaram moderada capacidade de sequestro do radical DPPH ao longo do tempo da reação. Os extratos de bacuri e tamarindo exibiram baixa capacidade de redução do radical DPPH durante os 20 minutos de reação

Os resultados da atividade antioxidante pelo método ABTS foram expressos como capacidade antioxidante total equivalente ao Trolox (valores TEAC). Constata-se, a partir da Tabela 3, que, assim como no teste antioxidante pelo método de captura de radicais DPPH', a polpa de acerola apresentou a mais elevada capacidade antioxidante, com valores TEAC de 1,605 $\pm 0,4264$ e 3,69 $\pm 0,209 \mathrm{mM}$ Trolox/g de polpa para os extratos aquosos e hidroalcoólicos, respectivamente, valores estatisticamente diferentes dos demais $(p<0,05)$. Com relação ao extrato aquoso das demais polpas de frutas, percebe-se que não houve diferença estatística entre as mesmas.

Quando confrontados os valores TEAC encontrados neste estudo para a polpa de acerola, com valores TEAC obtidos de outros alimentos de conhecido potencial antioxidante, como tomate $(1,65$ $\mathrm{mM} / \mathrm{g})$, azeite virgem de oliva $(1,79 \mathrm{mM} / \mathrm{mL})$, cerveja $(1,0 \mathrm{mM} / \mathrm{mL})$ e chá preto $(3,6 \mathrm{mM} / \mathrm{mL})$ (DE BEER et al., 2003), pode-se considerar a polpa de acerola como um alimento de elevada capacidade antioxidante.

Os extratos de polpas de bacuri e tamarindo apresentaram as menores atividades antioxidantes com TEAC de $0,094 \pm 0,003$ e $0,075 \pm 0,008 \mathrm{mM}$ de TROLOX/g de polpa de fruta, respectivamente, para o extrato aquoso, e TEAC de 0,052 $\pm 0,013 \mathrm{e}$ $0,114 \pm 0,019 \mathrm{mM}$ de TROLOX/g de polpa de fruta, respectivamente, para extrato hidroalcoólico.

Pode-se afirmar que, nesse estudo, houve uma relação direta entre o conteúdo de fenólicos totais e a capacidade antioxidante das polpas analisadas. As polpas que apresentaram os maiores conteúdos de fenólicos totais foram as que apresentaram a maior atividade antioxidante, tanto utilizando os radicais $\mathrm{DPPH}$, como os radicais ABTS. 
TABELA 1 - Teores de fenólicos em mg de ácido gálico por $100 \mathrm{~g}$ de polpa de fruta congelada.

\begin{tabular}{c|c|c}
\hline \multirow{2}{*}{ Polpa de Fruta } & \multicolumn{2}{|c}{ Fenólicos Totais (mg ácido gálico/ 100 g de polpa) } \\
\cline { 2 - 3 } & Extrato Aquoso & Extrato Hidroalcoólico \\
\hline Acerola & $835,25 \pm 32,44 \mathrm{a}$ & $449,63 \pm 10,24 \mathrm{a}$ \\
Bacuri & $10,35 \pm 0,42 \mathrm{~d}$ & $7,23 \pm 0,08 \mathrm{~d}$ \\
Cajá & $70,92 \pm 1,31 \mathrm{c}$ & $6,62 \pm 0,90 \mathrm{~d}$ \\
Caju & $201,61 \pm 19,15 \mathrm{~b}$ & $165,07 \pm 4,10 \mathrm{~b}$ \\
Goiaba & $104,76 \pm 4,39 \mathrm{c}$ & $20,21 \pm 1,95 \mathrm{c}$ \\
Tamarindo & $23,57 \pm 0,36 \mathrm{~d}$ & $23,35 \pm 0,21 \mathrm{c}$ \\
\hline
\end{tabular}

a,b,c,d médias, na mesma coluna, seguidas de letras diferentes diferem entre si, estatisticamente, de acordo com o teste de Tukey ( $\mathrm{p}<0,05)$.

TABELA 2 - Capacidade antioxidante $\left(\mathrm{EC}_{50} \mathrm{em} \mu \mathrm{g} / \mathrm{mL}\right)$ dos extratos aquoso e hidroalcóolico de polpas de frutas, utilizando o radical livre DPPH*.

\begin{tabular}{c|cc}
\hline \multirow{2}{*}{ Polpa de fruta } & \multicolumn{2}{|c}{$\mathrm{EC}_{50} \mathrm{em} \mu \mathrm{g} / \mathrm{mL}$} \\
\cline { 2 - 3 } & Extrato Aquoso & Extrato Hidroalcoólico \\
\hline Acerola & 24,42 & 1,74 \\
Bacuri & 4700,24 & 2356,96 \\
Cajá & 535,53 & 486,65 \\
Caju & 154,95 & 259,18 \\
Goiaba & 433,36 & 19,69 \\
Tamarindo & 2193,79 & 1431,47 \\
\hline
\end{tabular}

TABELA 3 - Valor TEAC (Capacidade Antioxidante Total Equivalente ao TROLOX) pelo método ABTS para os extratos aquosos e hidroalcóolico de polpa de fruta congelada.

\begin{tabular}{c|cc}
\hline \multirow{2}{*}{ Polpa de Fruta } & \multicolumn{2}{|c}{ Valor TEAC $(\mathrm{mM}$ TROLOX/g de polpa) } \\
\cline { 2 - 3 } & Extrato Aquoso & Extrato Hidroalcoólico \\
\hline Acerola & $1,605 \pm 0,426 \mathrm{a}$ & $3,690 \pm 0,209 \mathrm{a}$ \\
Bacuri & $0,094 \pm 0,003 \mathrm{~b}$ & $0,052 \pm 0,012 \mathrm{~d}$ \\
Goiaba & $0,198 \pm 0,026 \mathrm{~b}$ & $0,401 \pm 0,193 \mathrm{~b}$ \\
Cajá & $0,140 \pm 0,016 \mathrm{~b}$ & $0,219 \pm 0,113 \mathrm{c}$ \\
Caju & $0,212 \pm 0,022 \mathrm{~b}$ & $0,561 \pm 0,033 \mathrm{~b}$ \\
Tamarindo & $0,075 \pm 0,00^{8} \mathrm{~b}$ & $0,114 \pm 0,019 \mathrm{c}, \mathrm{d}$ \\
\hline
\end{tabular}

a,b,c,d médias, na mesma coluna, seguidas de letras diferentes diferem entre si estatisticamente $(\mathrm{p}<0,05)$.

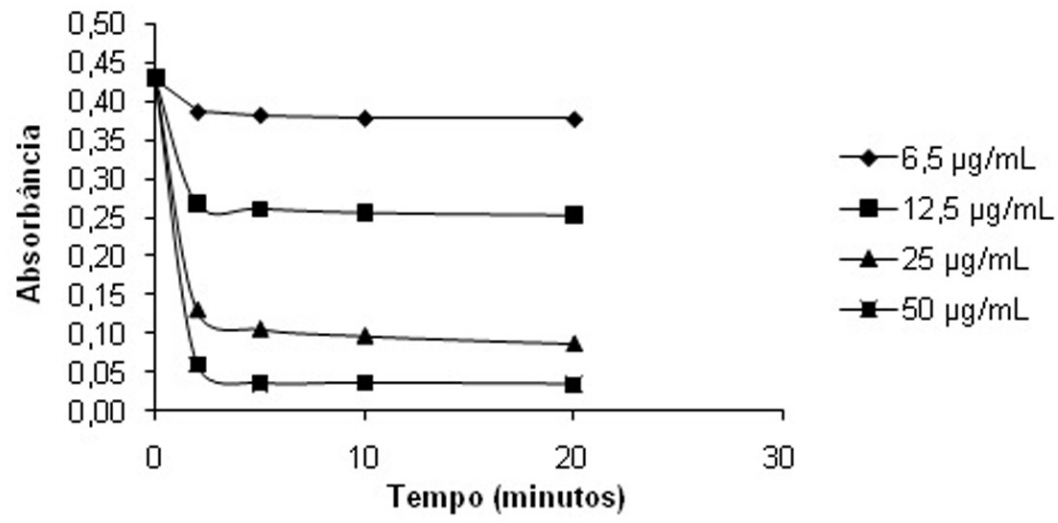

FIGURA 1 - Curva cinética do potencial antioxidante do extrato aquoso da polpa de acerola pelo método de DPPH. 

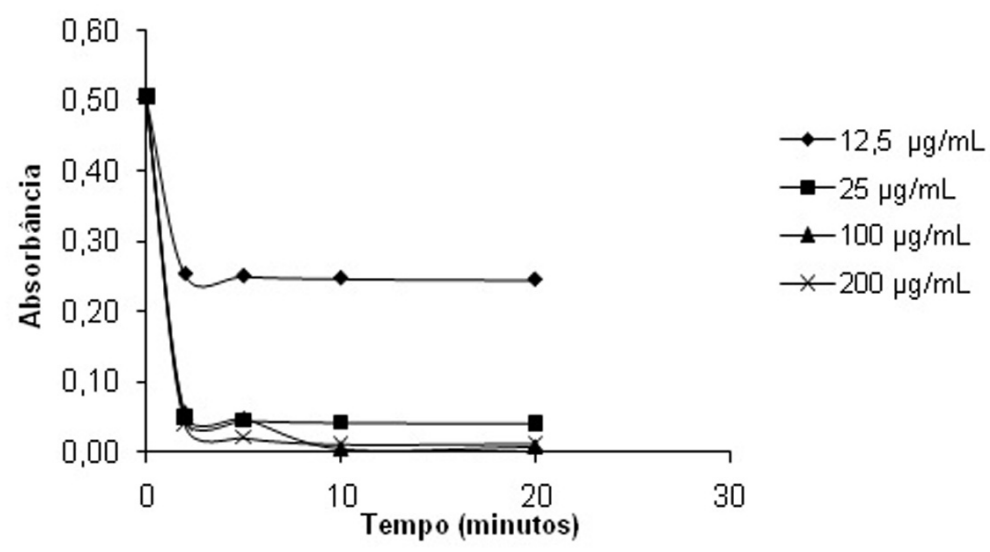

FIGURA 2 - Curva cinética do potencial antioxidante do extrato hidroalcoólico da polpa de acerola pelo método de DPPH.

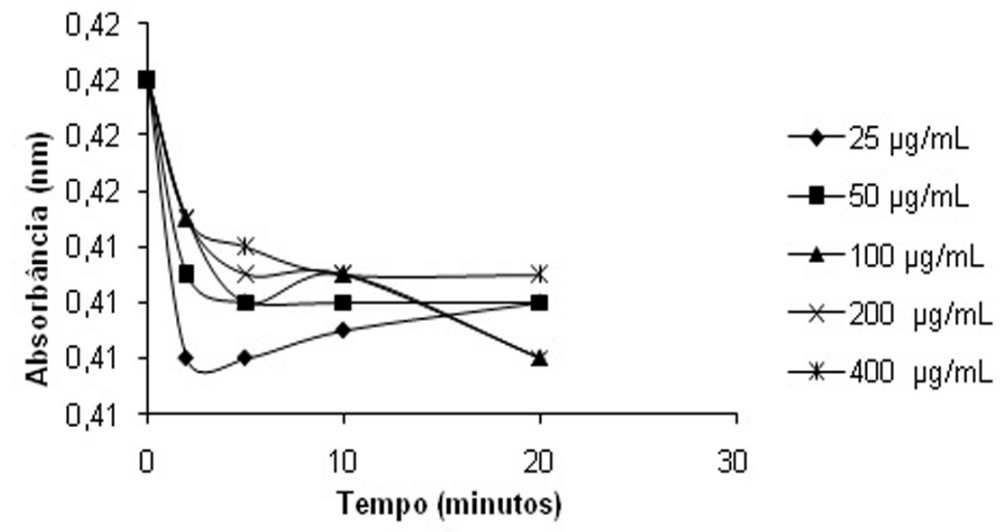

FIGURA 3 - Curva cinética do potencial antioxidante do extrato aquoso da polpa de bacuri pelo método de DPPH.

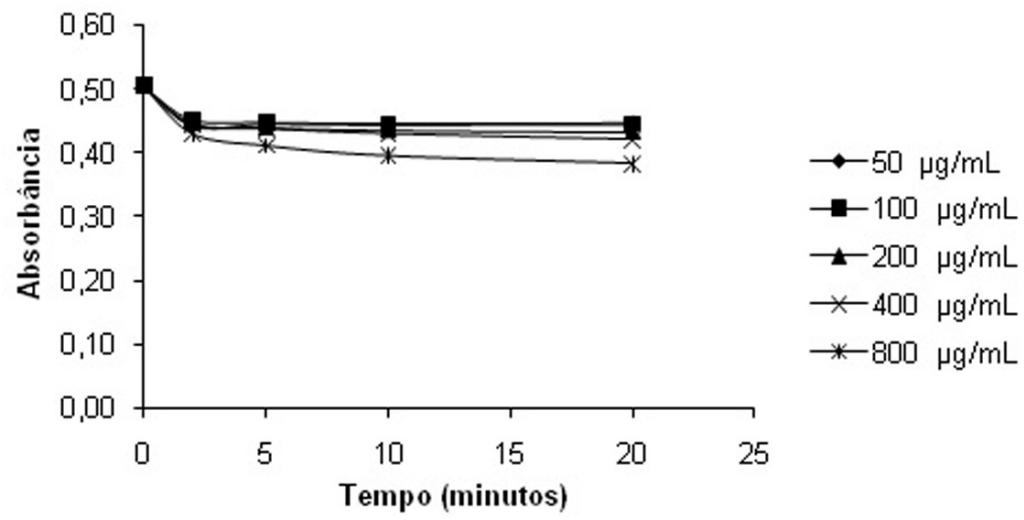

FIGURA 4 - Curva cinética do potencial antioxidante do extrato hidroalcoólico da polpa de bacuri pelo método de DPPH. 

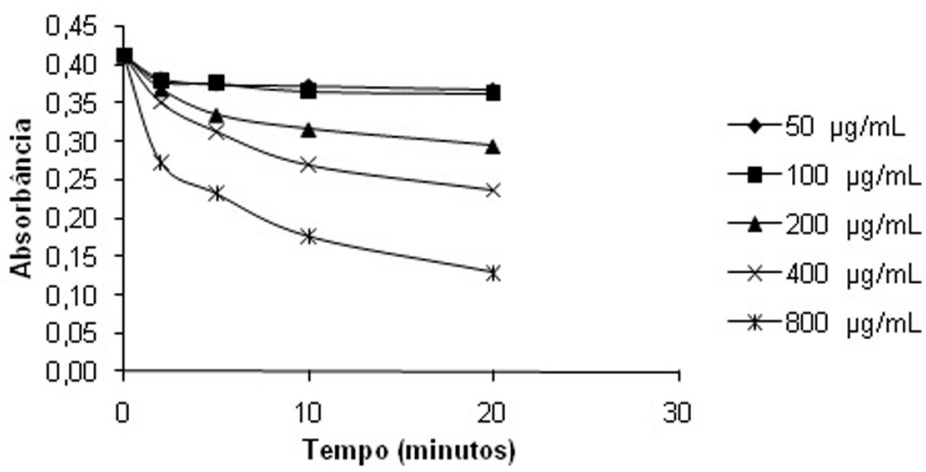

FIGURA 5 - Curva cinética do potencial antioxidante do extrato aquoso da polpa de cajá pelo método de $\mathrm{DPPH}$.

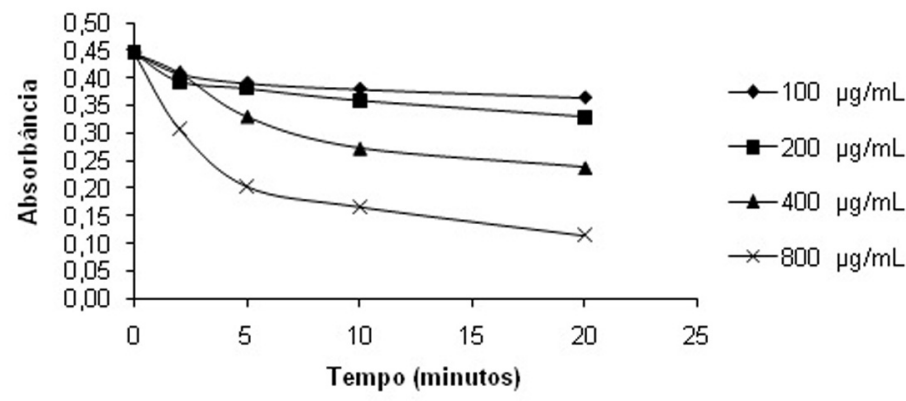

FIGURA 6 - Curva cinética do potencial antioxidante do extrato hidroalcoólico da polpa de cajá pelo método de DPPH.

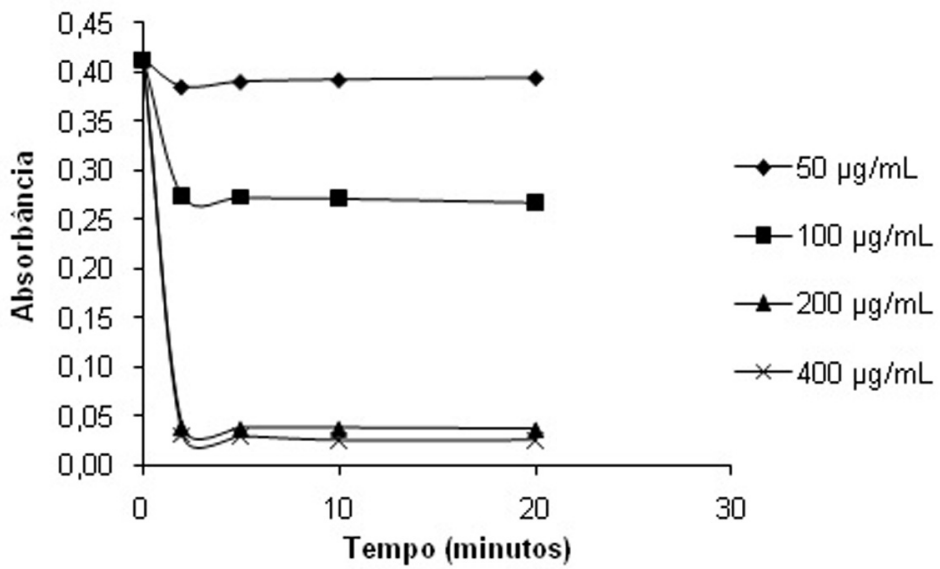

FIGURA 7 - Curva cinética do potencial antioxidante do extrato aquoso da polpa de caju pelo método de DPPH. 


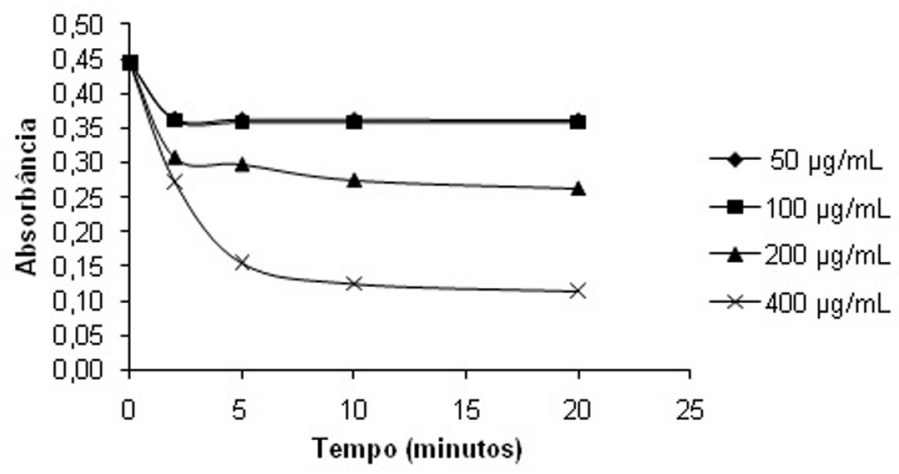

FIGURA 8 - Curva cinética do potencial antioxidante do extrato hidroalcoólico da polpa de caju pelo método de DPPH.

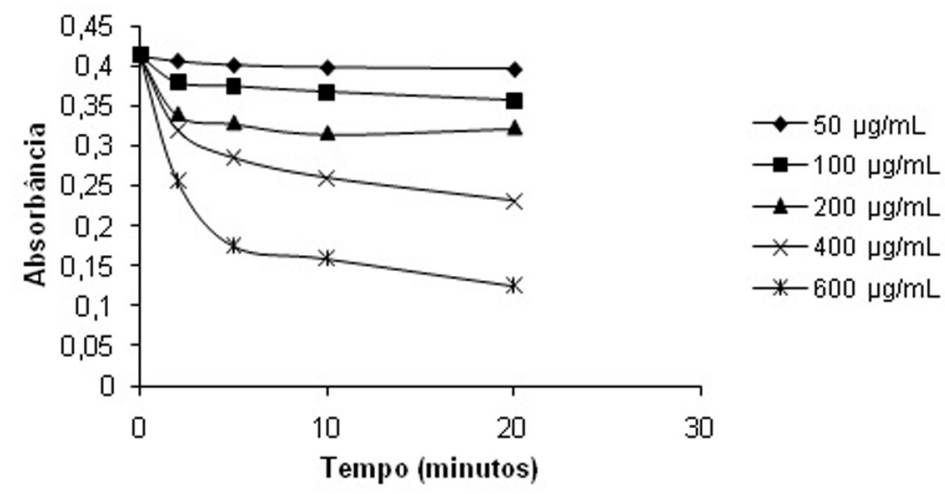

FIGURA 9 - Curva cinética do potencial antioxidante do extrato aquoso da polpa de goiaba pelo método de DPPH.

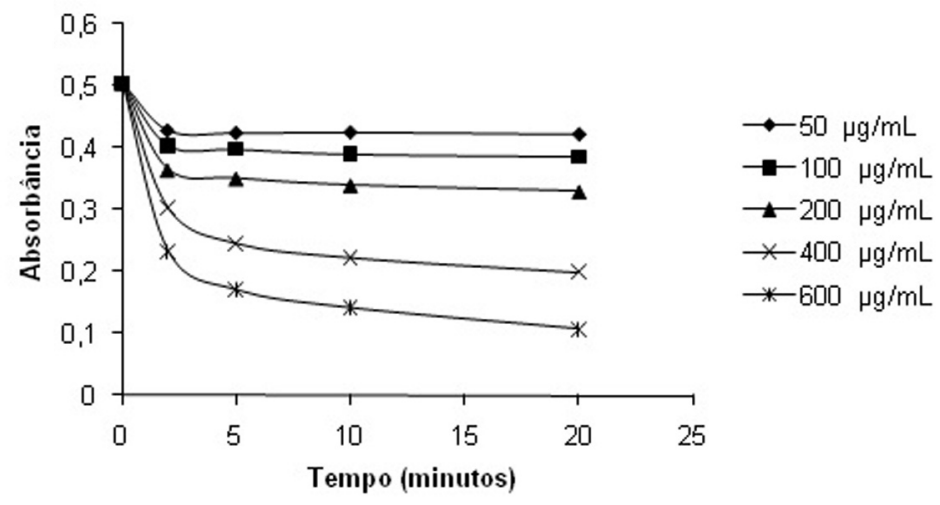

FIGURA 10 - Curva cinética do potencial antioxidante do extrato hidroalcoólico da polpa de goiaba pelo método de DPPH. 


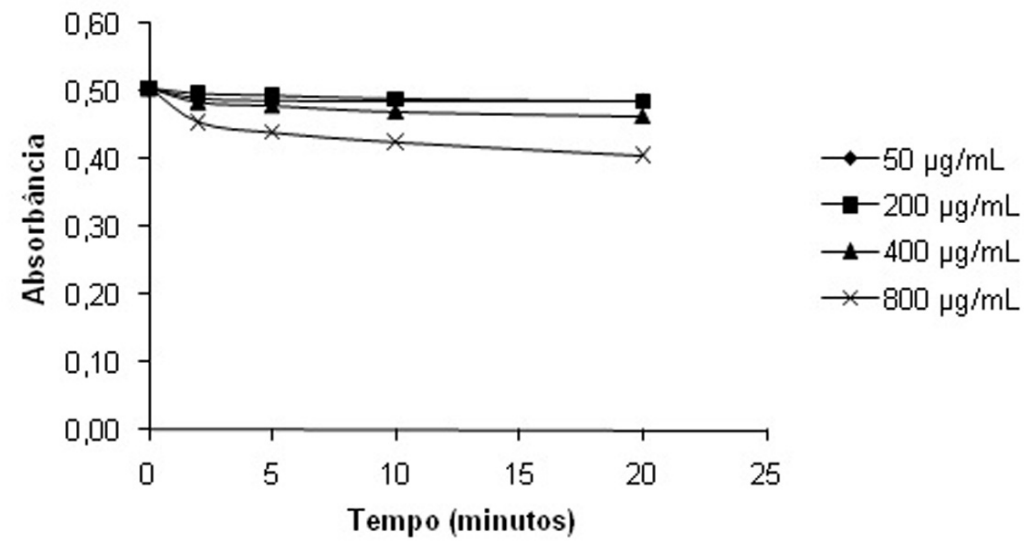

FIGURA 11 - Curva cinética do potencial antioxidante do extrato aquoso da polpa de tamarindo pelo método de DPPH.

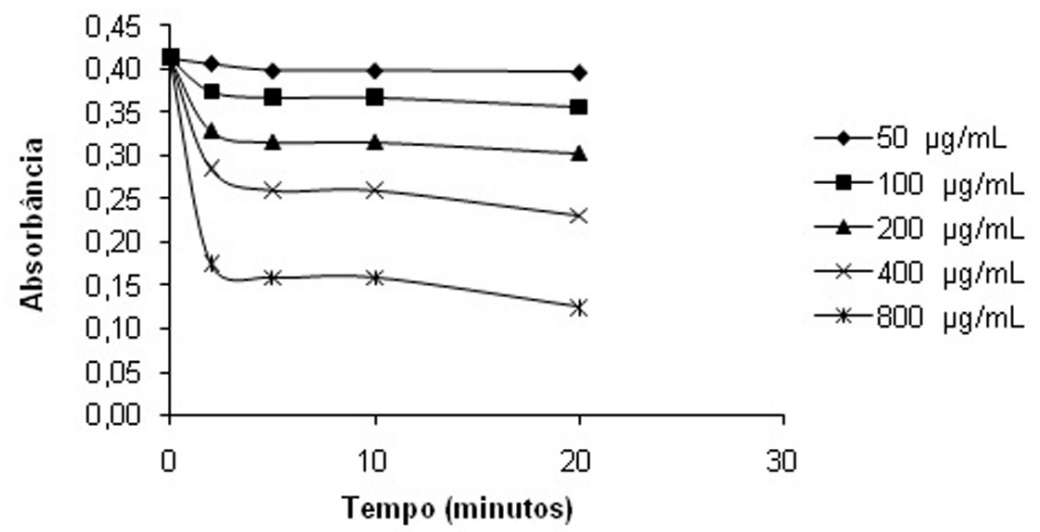

FIGURA 12 - Curva cinética do potencial antioxidante do extrato hidroalcoólico da polpa de tamarindo pelo método de DPPH.

\section{CONCLUSÃO}

O extrato de polpa de fruta que apresenta maior quantidade de compostos fenólicos é o de acerola, seguido pelo extrato de polpa de caju e de goiaba. Entre os valores de $\mathrm{EC}_{50}$ para a atividade antioxidante pelo método de captura de radicais DPPH, os obtidos com o extrato de polpa de acerola são os mais significativos, com apenas $1,74 \mu \mathrm{g} / \mathrm{mL}$ do extrato hidroalcoólico. Os valores TEAC mostram valores coerentes em relação à atividade antioxidante pelo método de DPPH e a quantificação de compostos fenólicos. Os extratos de polpa de acerola apresentam os melhores resultados, sendo assim, a de maior capacidade antioxidante dentre as demais polpas de frutas analisadas.

\section{REFERÊNCIAS}

BRAND-WILLIAMS, W.; CUVELIER, M.E.; BERSET, C. Use of a free radical method to evaluate antioxidant activity. Lebensmittel-Wissenschaft und-Technologie, London, v.28, n.1, p. 25-30, 1995.

DE BEER, D.; JOUBERT, E.; GELDERBLOM, W.C.A.; MANLEY, M. Antioxidant activity of south African red and white cultivar wines: free radical scavenger. Journal of Agricultural and Food Chemistry, Washington, v.90, p. 569-577, 2003. 
GONÇALVES, A. E. S. S. Avaliação da capacidade antioxidante de frutas e polpa de frutas nativas e determinação de teores de flavonoides e vitamina C. 2008. 88 f. Dissertação (Mestrado em Ciências dos Alimentos) - Faculdade de Ciências Farmacêuticas, Universidade de São Paulo, São Paulo, 2008.

HEIN, K.E.; TAGLIAFERRO A.R.; BOBILYA, D. J. Flavonoid antioxidants: Chemistry, metabolism and structure-activity relation ships. Journal of Nutritional Biochemistry, Stonehaum, v. 13, p. 572-584, 2002.

KUSKOSKI, E. A. et al. Frutos tropicais silvestres e polpas de frutas congeladas: atividade antioxidante, polifenóis e antocianinas. Ciência Rural, Santa Maria, v.36, n.4, p.1285-1286, 2006.

LIMA, A. de. Caracterização química, avaliação da atividade antioxidante in vitro $\mathrm{e}$ in vivo $\mathrm{e}$ identificação dos compostos fenólicos presentes no pequi (Caryocar brasiliense Camb.). 2008. 186 f. Tese (Doutorado em Bromatologia) - Faculdade de Ciências Farmacêuticas, Universidade de São Paulo, São Paulo, 2008.

MAIA, G. A.; SOUSA, P. H. M. S.; LIMA, A. S. Processamento de sucos de frutas tropicais. Fortaleza: Editora UFC, 2007. p 320.

MELO, E.A.; MACIEL, M.I.S.; LIMA, V.A.G.L.; NASCIMENTO, R.J. Capacidade antioxidante de frutas. Revista Brasileira de Ciências Farmacêuticas, São Paulo, v. 44, n.2, p. 193-201, 2008.

OLIVEIRA, M. E. B.; BASTOS, M. S. R.; FEITOSA, T.; BRANCO, M. A. A. C.; SILVA, M. G. G. Avaliação de parâmetros de qualidade físicoquímicos de polpas congeladas de acerola, cajá e caju. Ciência e Tecnologia de Alimentos, Campinas, v. 19, n.3, p. 326-332, 1999.

PÉREZ-JIMÉNEZ et al. Updated methodology to determine antioxidant capacity in plant foods, oils and beverages: Extraction, measurement and expression of results. Food Research International, Darking, v. 41, n. 3 , p. $274-285,2008$.
RE, R.; PELEGRINI, N.; PROTEGGENTE, A.; PANNALA, A.; YANG, M.; RICEEVANS, C. Antioxidant activity applying an improved ABTS radical cation decolorization assay. Free Radical Biology \& Medicine, New York, v.26, n.9-10, p.1231-1237, 1999.

SANTOS, C.A. do A.; COELHO, A.F.S.; CARREIRO, S. C. Avaliação microbiológica de polpas de frutas congeladas. Ciência e Tecnologia de Alimentos, Campinas, v. 28, n. 4, p. 913-915, 2008.

SOARES, M.; WELTER, L.; KUSKOSKI, E.M.; GONZAGA, L.; FETT, R. Compostos fenólicos e atividade antioxidante da casca de uvas niágara e isabel. Revista Brasileira de Fruticultura, Jaboticabal, v. 30, n. 1, p. 059-064, 2008.

SHAHIDI, F.; ALASALVAR, C.; LIYANA-PATHIRANA, C.M. Antioxidant phytochemicals in hazelnut kernel (Corylus avellana L.) and hazelnut byproducts. Journal of Agricultural and Food Chemistry, Washington, v.55, n.4, p.1212-1220, 2007.

SWAIN, T.; HILLS, W.E. The phenolic constituents of Punnus domestica. The quantitative analysis of phenolic constituents. Journal of the Science of Food and Agriculture, London, v.19, p. 63-68, 1959.

TEIXEIRA, C.G. A fruticultura no Brasil. Jornal da Cidade, Poços de Caldas, 2008.Disponível em: $<$ http://www.jorcidade.com.br/index. php?option $=$ com_content $\&$ view $=$ article $\& i d=38$ :qafruticultura-no-brasilq-por-cyro-goncalves-teixei ra\&catid $=13$ : todos \&Itemid $=19>$. Acesso em: 17 mar. 2009.

WU, X.; BEECHER, G.R.; HOLDEN,J.M.; HAYTOWITZ, D.B.; GEBHARDT, S.E.; PRIOR, R.L. Lipophilic and hidrophilic antioxidant capacities of common foods in the United States. Journal of Agricultural and Food Chemistry, Easton, v. 52, n.12, p. 4026-4037, 2004. 